\title{
A half-step in quantized conductance for low-density electrons in a quantum wire
}

\author{
Godfrey Gumbs ${ }^{1,4} *$ Antonios Balassis $2 \pm$ Danhong \\ Huang $^{3} \pm$ Sheehan Ahmed ${ }^{2} \S$ and Ryan Brennan 2 - \\ ${ }^{1}$ Department of Physics and Astronomy, \\ Hunter College of the City University of New York, \\ 695 Park Avenue, New York, NY 10065, USA \\ ${ }^{2}$ Physics Department, Fordham University, \\ 441 East Fordham Road, Bronx, NY 10458, USA \\ ${ }^{3}$ Air Force Research Laboratory, Space Vehicles Directorate, \\ Kirtland Air Force Base, NM 87117, USA and \\ ${ }^{4}$ Donostia International Physics Center (DIPC), P. de Manuel Lardizabal, \\ 4, 20018 San Sebastián, Basque Country, Spain
}

(Dated: August 8, 2018)

\begin{abstract}
We investigated the effect due to perpendicular magnetic field on quantum wires where spinorbit interaction (SOI) of electrons is not neglected. Based on the calculated energy dispersion, the nonlinear ballistic conductance $(G)$ and electron-diffusion thermoelectric power $\left(S_{d}\right)$ are calculated as functions of electron density, temperature and applied bias voltage. A low-temperature half-step feature in $G$, which was observed experimentally by Quay et al. [see Nature Physics 6, 336 (2010)], as well as a new peak in $S_{d}$ are reproduced here in the low density regime. These phenomena are related to the occurrence of the Zeeman splitting and SOI induced saddle point in the band structure, where the channel chemical potential lies within an anticrossing gap between the saddle point of the lower subband and the bottom of the upper subband. Additionally, side peaks in $G$ far away from the zero bias for the nonlinear transport, as well as a quadratic bias-voltage dependence of $G$ near zero voltage, are predicted and discussed.
\end{abstract}




\section{INTRODUCTION}

Quasi-one-dimensional (quasi-1D) electron systems (also called quantum wires) have attracted considerable attention both theoretically $\underline{\underline{1}}^{-\underline{\underline{3}}}$ and experimentally $\underline{\underline{4}} \underline{\underline{5}}$ since their experimental realization in $1986 . \underline{6}$ When an external magnetic field is applied in a direction perpendicular to a quantum wire, Landau quantization in such a system combines with lateral confinement, enhancing the quantum-wire confinement potential as well as the effective mass of electrons along the wire direction at the same time. $\stackrel{7.8}{\underline{*}}$ Several studies were reported for such systems, including magneto-optical properties $\frac{5,9}{9}$ and magneto-transport properties $\underline{10}-15$ in the diffusive regime. Moreover, the ballistic transport properties $\underline{16}-18$ of quantum wires were also explored under a tilted magnetic field.

On the other hand, the effect of Rashba spin-orbit interaction (SOI) on the ballistic and diffusive electron transport properties in quantum wires has also been investigated theoretically and experimentally in the absence of a magnetic field. $\underline{19}-\underline{23}$ Compared with the case when SOI is neglected, $\underline{\underline{24}}$ the quantum-wire conductance quantization in the presence of SOI is modified significantly at low temperatures. $\frac{23}{}$ Although the SOI-induced gap has been observed experimentally, $\underline{25}$ there are only a few theoretical studies dealing with ballistic electron transport and thermoelectric properties in parabolically-confined quantum wires with both SOI and a magnetic field. $\underline{26}-\underline{28}$

In this paper, we include the effects due to the edges of a realistic quantum wire through sharp and high potential walls forming the boundaries, instead of assuming a parabolic potential for the lateral confinement of the quantum-wire. Extending our previous study for zero magnetic field in the linear response regime, $\stackrel{23}{ }$ we calculate the bias-voltage dependent nonlinear ballistic conductance and electron-diffusion thermoelectric power for this quasione-dimensional system with a channel length less than the electron mean free path, where we have included the effect on the SOI of a finite magnetic field perpendicular to the quantum wire as well as the Zeeman effect on the electron spin states. A half-step feature in the quantized conductance is obtained for low electron densities and low temperatures.

The rest of this paper is arranged as follows. In Sec.II, we present our model for quantum wires with SOI under a perpendicular magnetic field. Numerical results and discussion of the energy bands, conductance and diffusion thermoelectric power as functions of electron 
density, temperature and bias voltage are presented in Sec.III. We summarize our results in Sec.IV,

\section{THE MODEL}

In the presence of a magnetic field $\mathbf{B}$ (in the $z$ direction) perpendicular to a quantum wire which is confined in the $x$ direction such that $|x| \leq \mathcal{W} / 2$ and extends along the $y$ direction, the spin-coupled Schrödinger equations for the electron wave-function spinor, $\left[\psi_{k_{y}}^{\uparrow}(x), \psi_{k_{y}}^{\downarrow}(x)\right]^{T}$, with the Landau gauge are written as $\underline{23}$

$$
\begin{gathered}
-\frac{\hbar^{2}}{2 m^{*}}\left[\frac{d^{2}}{d x^{2}}-\left(k_{y}+\frac{x}{\ell_{c}^{2}}\right)^{2}\right] \psi_{k_{y}}^{\uparrow}(x)+\alpha\left[\frac{d}{d x}+\left(k_{y}+\frac{x}{\ell_{c}^{2}}\right)\right] \psi_{k_{y}}^{\downarrow}(x) \\
-\beta \frac{\mathcal{W}}{\ell_{0}}\left(k_{y}+\frac{x}{\ell_{c}^{2}}\right) \mathcal{F}(x) \psi_{k_{y}}^{\uparrow}(x)=\left(\varepsilon-\frac{1}{2} g^{*} \hbar \omega_{c}\right) \psi_{k_{y}}^{\uparrow}(x), \\
-\frac{\hbar^{2}}{2 m^{*}}\left[\frac{d^{2}}{d x^{2}}-\left(k_{y}+\frac{x}{\ell_{c}^{2}}\right)^{2}\right] \psi_{k_{y}}^{\downarrow}(x)-\alpha\left[\frac{d}{d x}-\left(k_{y}+\frac{x}{\ell_{c}^{2}}\right)\right] \psi_{k_{y}}^{\uparrow}(x) \\
+\beta \frac{\mathcal{W}}{\ell_{0}}\left(k_{y}+\frac{x}{\ell_{c}^{2}}\right) \mathcal{F}(x) \psi_{k_{y}}^{\downarrow}(x)=\left(\varepsilon+\frac{1}{2} g^{*} \hbar \omega_{c}\right) \psi_{k_{y}}^{\downarrow}(x),
\end{gathered}
$$

where $\beta$ and $\alpha$ are the SOI parameters in the transverse $x$ and $z$ directions, respectively. Also, $\varepsilon$ is the eigenenergy of the electrons, the symbols $\uparrow$ and $\downarrow$ denote the up and down spin states, $k_{y}$ is the electron wave number along the wire, $m^{*}$ is the effective mass, $\omega_{c}=e B / m^{*}$ is the cyclotron frequency, $\ell_{c}=\sqrt{\hbar / e B}$ is the magnetic length, and $g^{*}$ is the effective Lande spin $g$-factor. Additionally, the quantum confinement factor ${ }^{23}$ introduced in the above equations for a non-parabolic confinement is given by

$$
\mathcal{F}(x)=\exp \left[-\frac{(x+\mathcal{W} / 2)^{2}}{2 \ell_{0}^{2}}\right]-\exp \left[-\frac{(x-\mathcal{W} / 2)^{2}}{2 \ell_{0}^{2}}\right],
$$

where $\ell_{0}$ describes the steepness of the confinement potential at the two edges of the wire. Compared with the case of $B=0, \underline{\underline{23}}$ we see that $k_{y}$ is replaced by $\left(k_{y}+x / \ell_{c}^{2}\right)$, as well as the appearance of a Zeeman splitting term, in Eqs. (11) and (2) when $B \neq 0$.

For ballistic electrons in a quantum wire, the charge $(n=0)$ and the heat $(n=1)$ currents are calculated as 24 


$$
\begin{aligned}
\mathcal{Q}^{(n)}\left(V_{b}\right) & =(-e)^{1-n} \frac{1}{2 \pi} \sum_{j} \int_{-\infty}^{\infty} d k_{y}\left(\varepsilon_{j k_{y}}-\mu_{\mathrm{c}}\right) v_{j k_{y}} \\
& \times\left[\theta\left(-v_{j k_{y}}\right) f_{j k_{y}}^{(\mathrm{R})}\left(1-f_{j k_{y}}^{(\mathrm{L})}\right)+\theta\left(v_{j k_{y}}\right) f_{j k_{y}}^{(\mathrm{L})}\left(1-f_{j k_{y}}^{(\mathrm{R})}\right)\right] .
\end{aligned}
$$

In this notation, $j=1,2, \cdots$ is a composite index labeling the spin-resolved subbands, $v_{j k_{y}}=(1 / \hbar) d \varepsilon_{j k_{y}} / d k_{y}$ is the group velocity of electrons in the $j$ th subband, $\theta(x)$ is the unit step function, $\mu_{\mathrm{c}}$ is the chemical potential of the quantum wire, $f_{j k_{y}}^{(s)}=\left\{1+\exp \left[\left(\varepsilon_{j k_{y}}-\right.\right.\right.$ $\left.\left.\left.\mu_{s}\right) / k_{B} T\right]\right\}^{-1}, T$ is the temperature of the system, $s=\mathrm{R}, \mathrm{L}$ denotes the right and left electrodes connected to two ends of a quantum wire in the $y$ direction, $\mu_{\mathrm{L}}=\mu_{\mathrm{c}}-e V_{\mathrm{b}} / 2$ and $\mu_{\mathrm{R}}=\mu_{\mathrm{c}}+e V_{b} / 2$ represent the chemical potentials of the left and right electrodes, satisfying $\mu_{\mathrm{R}}-\mu_{\mathrm{L}}=e V_{b}$ with $V_{b}$ being the external bias voltage. For fixed chosen linear electron density $n_{1 \mathrm{D}}$ and $T, \mu_{\mathrm{c}}$ can be calculated using the following constraint equation

$$
\sum_{j}\left\{\int_{-\infty}^{\infty} \frac{d k_{y}}{\exp \left[\left(\varepsilon_{j k_{y}}-\mu_{\mathrm{c}}\right) / k_{B} T\right]+1}\right\}-2 \pi n_{1 \mathrm{D}}=0 .
$$

Once the $\mathcal{Q}^{(n)}\left(V_{b}\right)$ in Eq. (4) has been calculated, the bias-dependent ballistic conductance $G\left(V_{b}\right)$ of the quantum wire is simply given by

$$
G\left(V_{b}\right)=\frac{\mathcal{Q}^{(0)}\left(V_{b}\right)}{V_{b}},
$$

and the diffusion thermoelectric power $S_{\mathrm{d}}\left(V_{b}\right)$ may be calculated according to

$$
S_{\mathrm{d}}\left(V_{b}\right)=\frac{1}{T}\left[\frac{\mathcal{Q}^{(1)}\left(V_{b}\right)}{\mathcal{Q}^{(0)}\left(V_{b}\right)}\right] .
$$

We now employ these quantities to carry out our numerical calculations.

\section{NUMERICAL RESULTS}

In our calculations, we define two spin-related characteristic lengths, namely, $\ell_{\alpha}=$ $\hbar^{2} /\left(2 m^{*} \alpha\right)$ and $\ell_{\beta}=\hbar^{2} /\left(2 m^{*} \beta\right)$. Consequently, four corresponding dimensionless quantities related to spin-orbit coupling, quantum confinement factor and magnetic-field effect are given by: $\tau_{\alpha}=\mathcal{W} / \ell_{\alpha} \propto \alpha, \tau_{\beta}=\mathcal{W} / \ell_{\beta} \propto \beta, \tau_{0}=\mathcal{W} / \ell_{0}$ and $\tau_{c}=\mathcal{W} / \ell_{c} \propto \sqrt{B}$. The scaled electron kinetic energy is $\varepsilon_{j k_{y}} / E_{0}$ with $E_{0}=\pi^{2} \hbar^{2} / 2 m^{*} \mathcal{W}^{2}$, the scaled electron wave 
number is $k_{y} \mathcal{W}$, and the scaled position variable is $x / \mathcal{W}$. Parameters in our calculations were chosen as $m^{*}=0.067 m_{0}$ (as in GaAs) with $m_{0}$ being the free-electron mass, $g^{*}=-0.44$ due to exchange interaction, $\tau_{\beta}=10, \mathcal{W}=568.7 \AA$ and $\tau_{0}=10^{3}$. Other parameters, such as $\tau_{\alpha}, B, T, V_{b}$ and $n_{1 \mathrm{D}}$, will be given in the figure captions.

Figure 1 1 shows the calculated eigenenergy $\varepsilon_{j k_{y}} / E_{0}$ as a function of $k_{y} \mathcal{W}$ for three different cases, i.e., (a) $\tau_{\alpha}=10, B=0 \mathrm{~T}$; (b) $\tau_{\alpha}=10, B=1 \mathrm{~T}$; (c) $\tau_{\alpha}=0, B=1 \mathrm{~T}$. From Fig.11(a), we find that the spin-orbit interaction under $\alpha \neq 0$ couples spin-up and spin-down electron states in the absence of a $B$ field. Here, the energy dispersion curves of the spin-up and spin-down states are shifted relative to each other with respect to $k_{y}=0$. In the presence of a $B$ field, as shown in Fig.1(b), many anticrossing gaps are created due to both Zeeman splitting and SOI, the effective mass of electrons along the wire is enhanced, and new saddle points are formed. $\stackrel{13}{ }$ After the spin-orbit interaction is turned off, we simply see a set of Zeeman split parabolas, as demonstrated by Fig.1(c). When $B \rightarrow \infty$, these subbands will convert into discrete Landau levels (with infinite effective mass along the $y$ direction).

The results for the quantum-wire chemical potential $\mu_{\mathrm{c}} / E_{0}$, the ballistic conductance $G$ [in units of $\left.\left(e^{2} / h\right)\right]$, and the diffusion thermoelectric power $S_{\mathrm{d}}$ [in units of $\left(-k_{B} / e\right)$ ] are compared in Figs.2(a), (b) and (c), respectively, for the three cases discussed in Fig.11. Here, $T=4 \mathrm{~K}$ and a small bias voltage $V_{b}=0.1 \mathrm{meV}$ is used for the linear-response regime. From Fig.2(a) we see that when $\alpha \neq 0, \mu_{\mathrm{c}}$ is reduced at high electron densities but enhanced at low densities by the $B$ field due to the formed anticrossing gap and increased electron effective mass. Additionally, there exists a series of kinks in $\mu_{\mathrm{c}}$ as a function of $n_{1 \mathrm{D}}$, which is related to the onset of a new subband population. By turning off the SOI, $\mu_{\mathrm{c}}$ is increased for finite values of $B$ and the kinks in $\mu_{\mathrm{c}}$ are shifted at the same time due to the suppression of the flattened region around $k_{y}=0$ where the density of states is expected to be high. This series of kinks observed in Fig.2(a) leads to the occurrence of quantum steps for $G$ in Fig.2(b). Furthermore, each quantum step in $G$ relates to a peak in $S_{\mathrm{d}}$, as presented in Fig.2(c). It is interesting to note that the application of the $B$ field has a very profound effect on the spin-orbit interaction of electrons at low densities, where a new step (indicated by a black arrow) in (b) and a new peak in (c) are seen in correspondence to $\mu_{\mathrm{c}}$ lying inside the lowest anticrossing gap. Similar half-step features were observed experimentally. $\underline{25}$

Comparisons for $\mu_{\mathrm{c}}, G$ and $S_{\mathrm{d}}$ as a function of temperature $T$ for the three cases discussed 
in Fig.11are presented in Figs. 33(a), (b) and (c), respectively, where we chose $n_{1 \mathrm{D}}=10^{6} \mathrm{~cm}^{-1}$ and $V_{b}=0.1 \mathrm{meV}$. With a finite SOI, the $B$ field reduces (increases) $\mu_{\mathrm{c}}$ in Fig.3(a) for $T<4 \mathrm{~K}(T>4 \mathrm{~K})$. For $\alpha=0, \mu_{\mathrm{c}}$ is significantly enhanced. Meanwhile, $\mu_{\mathrm{c}}$, which lies within the gap of the second and third parabolas, increases with $T$ in contrast to the case when $\alpha \neq 0$ where a decreasing $\mu_{\mathrm{c}}$ with $T$ is obtained due to the fact that $\mu_{\mathrm{c}}$ stays very close to the two minima of the third subband in Figs.1(a) and (b). For nonzero SOI, $G$ increases with $T$ in Fig. 3(b) as a result of the gradual thermal population of higher subbands slightly above $\mu_{\mathrm{c}}$. On the other hand, $G$ is decreased when $T$ is increased in the absence of spin-orbit coupling since $G \propto\left[-\partial f_{j k_{y}}^{(s)} / \partial \varepsilon_{j k_{y}}\right]$ whose peak strength is reduced by increasing $T$ provided $\mu_{\mathrm{c}}$ lies within a gap. In addition, as can be seen from Fig.33(c), $S_{\mathrm{d}}$ monotonically increases with $T$, and becomes linearly proportional to $T$ above $4 \mathrm{~K} . \underline{14}$ However, it becomes exponentially small for $T \ll 1 \mathrm{~K} . \underline{14}$ For $\alpha=0, S_{\mathrm{d}}$ becomes much smaller than in the previous two cases due to a larger value of $G$.

We present in Fig. 4 comparisons between the bias voltage $\left(V_{b}\right)$ dependence of $G$ in (a), the low-voltage blow-out view of Fig. 4 (a) in (b) and the charge current $\mathcal{Q}^{(0)}$ in (c) for the three cases considered in Fig.1, where $n_{1 \mathrm{D}}=10^{6} \mathrm{~cm}^{-1}$ and $T=4 \mathrm{~K}$. When $\tau_{\alpha}=10$ in Fig.4(a), $G$ initially increases with $\left|V_{b}\right|$ from $2 e^{2} / h$ in a symmetric way, which is followed by a decrease before it increases again. The $B$ field does not alter the shape of the $G-V_{b}$ curve except for a small reduction of $G$. On the other hand, $G$ decreases with $\left|V_{b}\right|$ from $4 e^{2} / h$ and eventually saturates at $3 e^{2} / h$ for $\tau_{\alpha}=0$. These dramatically distinct behaviors in $G$ are attributed to different positions of the chemical potentials in the two electrodes relative to the bottom of subbands as $V_{b} \rightarrow 0$, as explained in Fig. 3. In order to see the behavior of $G$ in the low-voltage regime, we display in Fig. 4(b) the blow-out view of Fig. 4(a), where a quadratic dependence of $G$ on $V_{b}$ can be identified. Figure 4(c) exhibits a different dependence of $\mathcal{Q}^{(0)}$ on $V_{b}$. For the cases with $\tau_{\alpha}=10$, the leading term in the charge current is $\mathcal{Q}^{(0)} \propto V_{b}\left[-\partial f_{j k_{y}}^{(s)} / \partial \varepsilon_{j k_{y}}\right]$ for $\left|V_{b}\right| \leq 1.5 \mathrm{meV}$. However, for $\left|V_{b}\right| \geq 2.5 \mathrm{meV}$ in Fig. $4(c)$, we find that the leading term changes to $\mathcal{Q}^{(0)} \propto V_{b}^{3}\left[-\partial^{3} f_{j k_{y}}^{(s)} / \partial \varepsilon_{j k_{y}}^{3}\right]$. This explains the observations in Fig. 4 (b), i.e., $G=2 e^{2} / h$ for $V_{b} \rightarrow 0$ (linear response) and $G \propto V_{b}^{2}$ (nonlinear response) for large values of $\left|V_{b}\right|$. As $\tau_{\alpha}=0$, the slope in the linear-response regime is greatly enhanced because of a larger value of the Fermi velocity (large $\mu_{\mathrm{c}}$ ), leading to a quadratic decrease of $G$ with $V_{b}$ in Fig.4(b) as $\left|V_{b}\right|$ increases. 
In Fig. 5 we display two-dimensional (2D) contour plots of $G\left(n_{1 \mathrm{D}}, T\right)$ in (a) and (b) as well as $2 \mathrm{D}$ contour plots of $S_{\mathrm{d}}\left(n_{1 \mathrm{D}}, T\right)$ in (c) and (d) when $B=0[(\mathrm{a})$ and (c)] and when $B=1 \mathrm{~T}[(\mathrm{~b})$ and $(\mathrm{d})]$. We chose $V_{b}=0.1 \mathrm{meV}$ and $\tau_{\alpha}=10$. In general, for both $B=0$ and $B=1 \mathrm{~T}$, we clearly see quantized steps in $G$ and peaks in $S_{\mathrm{d}}$ with increasing $n_{1 \mathrm{D}}$ at any value of $T$ in this figure. The step and peak positions are almost independent of $T$. However, in the low-density regime when $n_{1 \mathrm{D}} \leq 0.5 \times 10^{6} \mathrm{~cm}^{-1}$, the presence of the $B$ field modifies

the spin-orbit coupling and dramatically alters the behaviors of $G$ and $S_{\mathrm{d}}$ as functions of both $n_{1 \mathrm{D}}$ and $T$.

We compare the $2 \mathrm{D}$ contour plots of $G\left(T, V_{b}\right)$ in Fig. 6 at $B=0$ in (a) and $B=1 \mathrm{~T}$ in (b), for chosen $n_{1 \mathrm{D}}=10^{6} \mathrm{~cm}^{-1}$ and $\tau_{\alpha}=10$. It is easy to see that the two strong peaks in $G$ on either side of $V_{b}=0$ are greatly suppressed by the magnetic field, which is further accompanied by an expanded dip around $V_{b}=0$. In addition, the peaks in $G$ decrease with $T$.

For completeness, we present in Fig. 7 the $2 \mathrm{D}$ contour plots of $G\left(n_{1 \mathrm{D}}, V_{b}\right)$ for $B=0$ and $B=1 \mathrm{~T}$ in (a) and (b), respectively, at $T=4 \mathrm{~K}$ when $\tau_{\alpha}=10$. It is clear that the quantized steps in $G$ become most observable close to $V_{b}=0$. The $B$ field does not significantly affect $G$ in the high-density regime $\left(n_{1 \mathrm{D}} \geq 2.5 \times 10^{6} \mathrm{~cm}^{-1}\right)$. In the intermediate-density regime $\left(1.2 \times 10^{6} \mathrm{~cm}^{-1} \leq n_{1 \mathrm{D}} \leq 2.5 \times 10^{6} \mathrm{~cm}^{-1}\right), G$ only weakly depends on $\left|V_{b}\right|$ and shows no $B$-field effect. In the low-density regime $\left(n_{1 \mathrm{D}} \leq 1.2 \times 10^{6} \mathrm{~cm}^{-1}\right)$, however, the $B$ field changes $G$ substantially as functions of both $n_{1 \mathrm{D}}$ and $\left|V_{b}\right|$. The dip around $V_{b}=0$ evolves into a peak and vice versa with increasing $n_{1 \mathrm{D}}$ due to the variation of the relative position of $\mu_{\mathrm{c}}$ with respect to the bottom of the subband.

\section{SUMMARY AND CONCLUDING REMARKS}

In summary, we have investigated the combined effect of SOI and an external magnetic field which is applied perpendicularly to a quantum wire. The quantities calculated are the the quasi-one-dimensional energy subbands, the nonlinear ballistic conductance $(G)$ and electron-diffusion thermoelectric power $\left(S_{d}\right)$ as functions of electron density, temperature and applied bias voltage. An important outcome of our numerical calculations is the observation of a half-step in the low-temperature quantized conductance at low electron densities. We have accounted for this feature as being due to the occurrence of a saddle point in the 
subband energy dispersion relation. The saddle point is due to the combined action of the applied magnetic field and spin-orbit coupling in the wire. A similar low-temperature halfstep feature in $G$ was observed experimentally by Quay et al. $\frac{25}{}$. A new peak in $S_{d}$ is produced in the low density regime, and is also related to the occurrence of the magnetic field and SOI induced saddle point in the band structure, where the channel chemical potential lies within an anticrossing gap between the saddle point of the lower subband and the bottom of the upper subband. Additionally, the present nonlinear transport theory has predicted side peaks in the quantized conductance as a function of bias voltage away from zero and a

quadratic bias dependence near zero voltage. This extension of our previous work ${ }^{23}$ clearly demonstrates that the application of magnetic field leads to observable differences in the ballistic transport properties of a quantum wire structure with SOI playing a role.

\section{Acknowledgments}

This research was supported by contract \# FA 9453-11-01-0263 of AFRL. DH would like to thank the Air Force Office of Scientific Research (AFOSR) for its support.

* Electronic address: ggumbs@hunter.cuny.edu

$\dagger$ Electronic address: balassis@fordham.edu

$\ddagger$ Electronic address: danhong.huang@kirtland.af.mil

$\S$ Electronic address: ashahmed@fordham.edu

ศ Electronic address: rybrennan@fordham.edu

1 Q. P. Li and S. Das Sarma, Phys. Rev. B 43, 11768 (1991).

2 G. Gumbs, D. H. Huang, Y. Yin, H. Qiang, D. Yan, F. H. Pollak and T. F. Noble, Phys. Rev. B 4818328 (1993).

3 G. Gumbs and D. H. Huang, Properties of Interacting Low-Dimensional Systems, (Wiley-VCH Verlag GmbH \& Co. KGaA, Weinheim, Germany, 2011), p. 275 and p. 303.

4 W. Hansen, M. Horst, J. P. Kotthaus, U. Merkt, Ch. Sikorski and K. Ploog, Phys. Rev. Lett. 58, 2586 (1987).

5 T. Demel, D. Heitmann, P. Grambow and K. Ploog, Phys. Rev. B 38, 12732 (1988). 
6 T. J. Thornton, M. Pepper, H. Ahmed, D. Andrews, and G. J. Davies, Phys. Rev. Lett. 56, $1198(1986)$.

7 H. L. Stormer, L. N. Pfeiffer, K. W. Baldwin, K. W. West and J. Spector, Appl. Phys. Lett. 58, 726 (1991).

8 G. Gumbs, D. H. Huang and D. Heitmann, Phys. Rev. B 44, 8084 (1991).

9 D. H. Huang, G. Gumbs and N. J. M. Horing, Phys. Rev. B 49, 11463 (1994).

10 D. Weiss, K. von Klitzing, K. Ploog and G. Weimann, Europhys. Lett. 8, 179 (1989).

11 C. Zhang and R. R. Gerhardts, Phys. Rev. B 4112850 (1990).

12 D. H. Huang and S. K. Lyo, J. Phys.: Condens. Matter 12, 3383 (2000).

13 S. K. Lyo and D. H. Huang, Phys. Rev. B 64115320 (2001).

14 S. K. Lyo and D. H. Huang, Phys. Rev. B 66155307 (2002).

15 S. K. Lyo and D. H. Huang, Phys. Rev. B 68115317 (2003).

16 S. F. Fischer, G. Apetrii, U. Kunze, D. Schuh and G. Abstreiter, Nat. Phys. 2, 91 (2006); Phys. Rev. B 74, 115324 (2006).

17 Lev G. Mourokh, A. Yu. Smirnov and S. F. Fischer, Appl. Phys. Lett. 90, 132108 (2007).

18 D. H. Huang, S. K. Lyo, K. J. Thomas and M. Pepper, Phys. Rev. B 77, 085320 (2008).

19 A. V. Moroz, K. V. Samokhin and C. H. W. Barnes, Phys. Rev. Lett. 84, 4164 (2000).

20 A. V. Moroz and C. H. W. Barnes, Phys. Rev. B 60, 14272 (1999).

21 Y. V. Pershin, J. A. Nesteroff, and V. Privman, Phys. Rev. B 69, 121306 (2004).

22 G. Gumbs, Phys. Rev. B 70, 235314 (2004).

23 G. Gumbs, A. Balassis and D. H. Huang, J. Appl. Phys. 108, 093704 (2010).

24 S. K. Lyo and D. H. Huang, J. Phys.: Condens. Matter 16, 3379 (2004).

25 C. H. L. Quay, T. L. Hughes, J. A. Sulpizio, L. N. Pfeiffer, K. W. Baldwin, K. W. West, D. Goldhaber-Gordon, and R. de Picciotto, Nat. Phys. 6, 336 (2010).

26 S. Debald and B. Kramer, Phys. Rev. B 71, 115322 (2005).

27 T.-Y. Zhang, W. Zhao and X.-M. Liu, J. Phys.: Condens. Matter 21, 335501 (2009).

28 H.-F. Lü, L.-C. Zhu, X.-T. Zu and H.-W. Zhang, Appl. Phys. Lett. 96, 123111 (2010). 

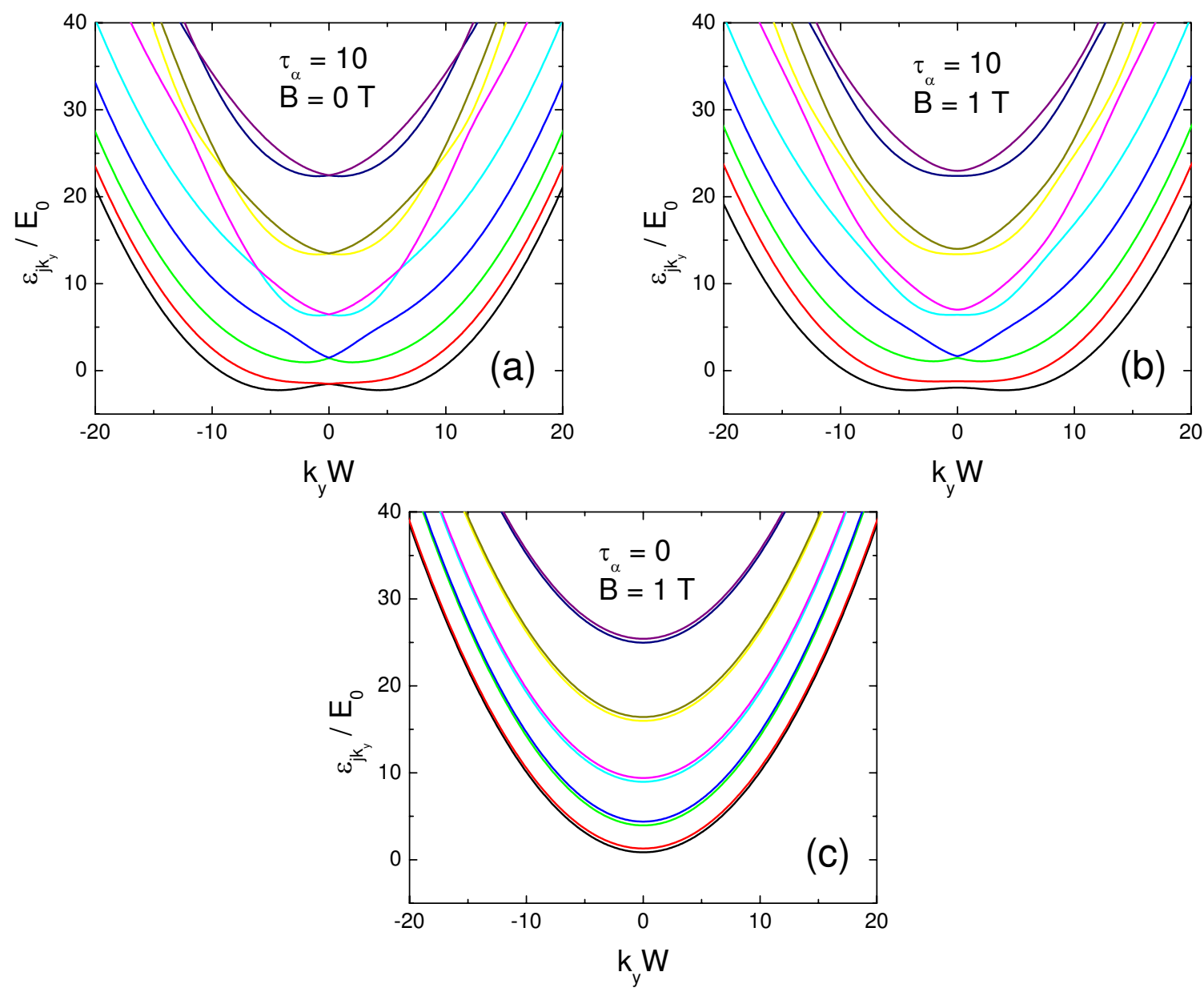

FIG. 1: (Color online) The calculated eigenenergies $\varepsilon_{j k_{y}} / E_{0}$ as functions of $k_{y} \mathcal{W}$ for the three cases considered: (a) $\tau_{\alpha}=10$ and $B=0 \mathrm{~T}$; (b) $\tau_{\alpha}=10$ and $B=1 \mathrm{~T}$; (c) $\tau_{\alpha}=0$ and $B=1 \mathrm{~T}$. The Zeeman splitting in (c) can be resolved for $B=1 \mathrm{~T}$. The degeneracy of $\uparrow$ and $\downarrow$ spin states at $k_{y}=0$ are split by the Zeeman effect in (b) to form an anticrossing gap. 

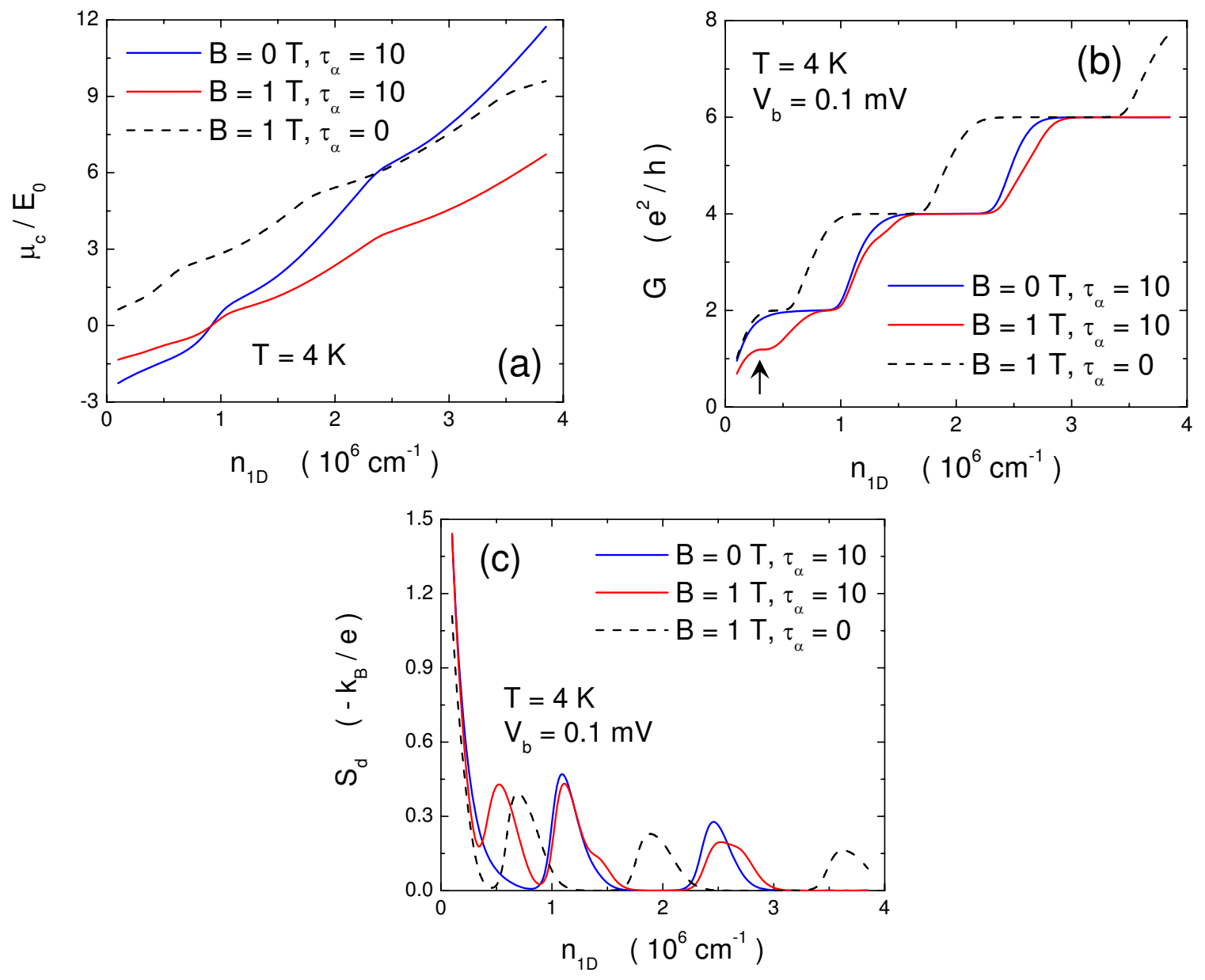

FIG. 2: (Color online) (a) Chemical potential $\mu_{\mathrm{c}}$ (in units of $E_{0}$ ) for a quantum wire, (b) ballistic conductance $G$ (in units of $\left.\left(e^{2} / h\right)\right)$ and (c) diffusion thermoelectric power $S_{d}$ (in units of $\left(-k_{B} / e\right)$ ) as functions of the linear density $n_{1 \mathrm{D}}$ for the three cases: $\tau_{\alpha}=10, B=0 \mathrm{~T}$ (blue solid curves); $\tau_{\alpha}=10, B=1 \mathrm{~T}$ (red solid curves); and $\tau_{\alpha}=0, B=1 \mathrm{~T}$ (black dashed curves), where $T=4 \mathrm{~K}$ and $V_{b}=0.1 \mathrm{meV}$. The black arrow in (b) indicates a half-step in quantized conductance $G$. 

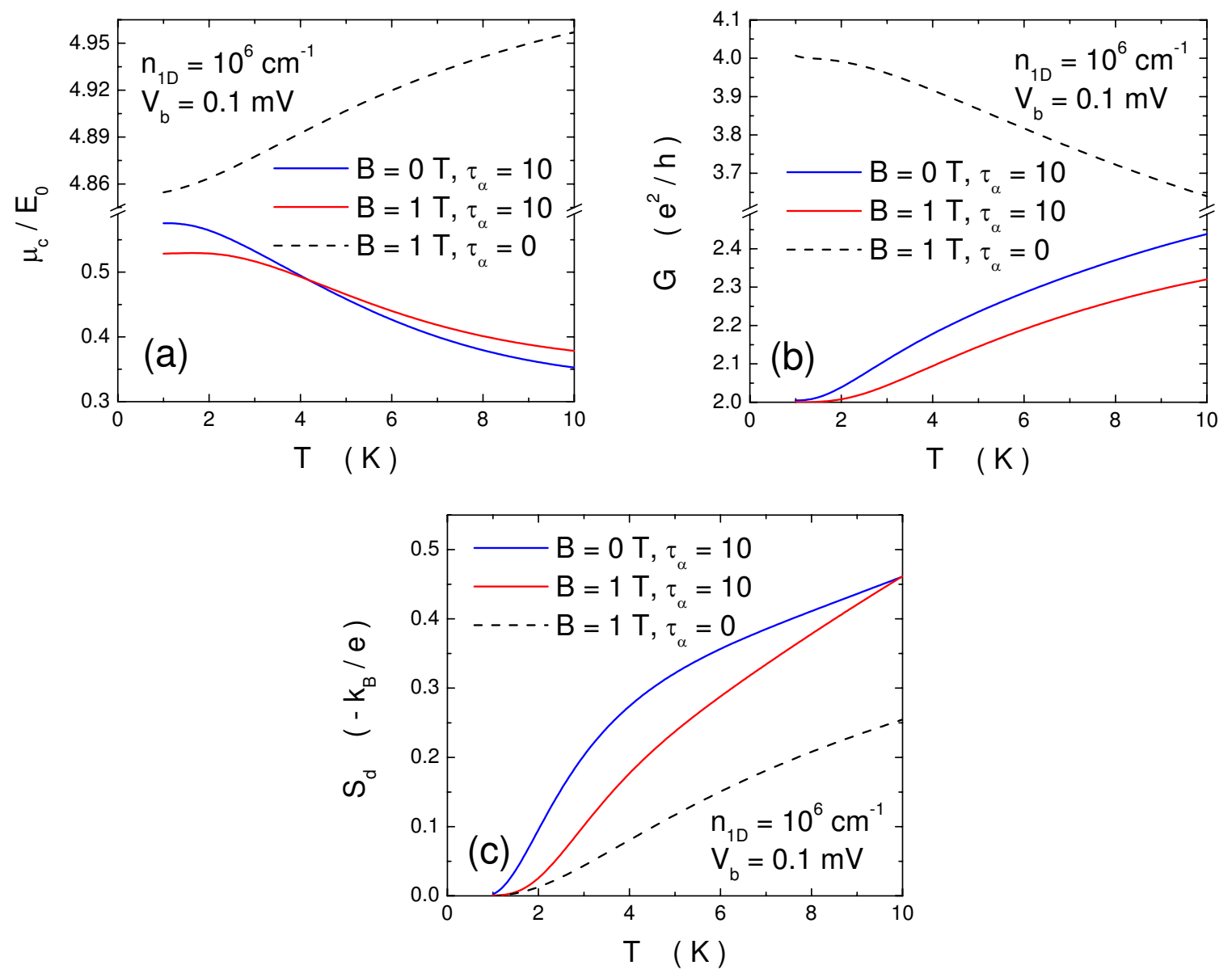

FIG. 3: (Color online) Calculated (a) $\mu_{\mathrm{c}}$, (b) $G$ and (c) $S_{d}$ as functions of temperature $T$ for the three cases: $\tau_{\alpha}=10, B=0 \mathrm{~T}$ (blue solid curves); $\tau_{\alpha}=10, B=1 \mathrm{~T}$ (red solid curves); and $\tau_{\alpha}=0$, $B=1 \mathrm{~T}$ (black dashed curves), where $n_{1 \mathrm{D}}=10^{6} \mathrm{~cm}^{-1}$ and $V_{b}=0.1 \mathrm{meV}$. 

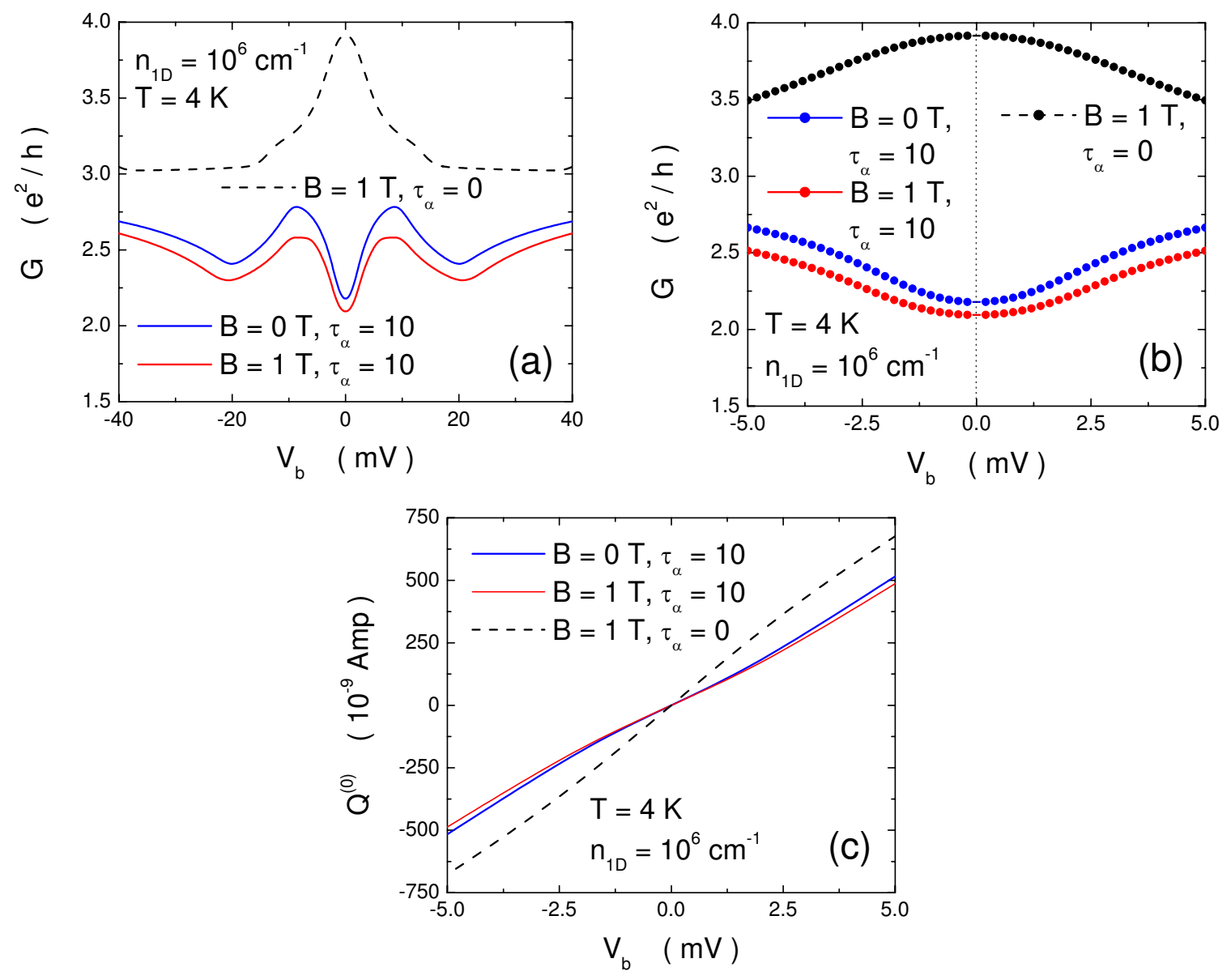

FIG. 4: (Color online) Calculated (a) G, (b) its blow-out view and (c) charge current $\mathcal{Q}^{(0)}$ as functions of the bias voltage $V_{b}$ for $\tau_{\alpha}=10, B=0 \mathrm{~T}$ (blue solid curves), $\tau_{\alpha}=10, B=1 \mathrm{~T}$ (red solid curves), and $\tau_{\alpha}=0, B=1 \mathrm{~T}$ (black dashed curves). Here, $T=4 \mathrm{~K}$ and $n_{1 \mathrm{D}}=10^{6} \mathrm{~cm}^{-1}$. The vertical dotted line in (b) indicates the position for $V_{b}=0$. 

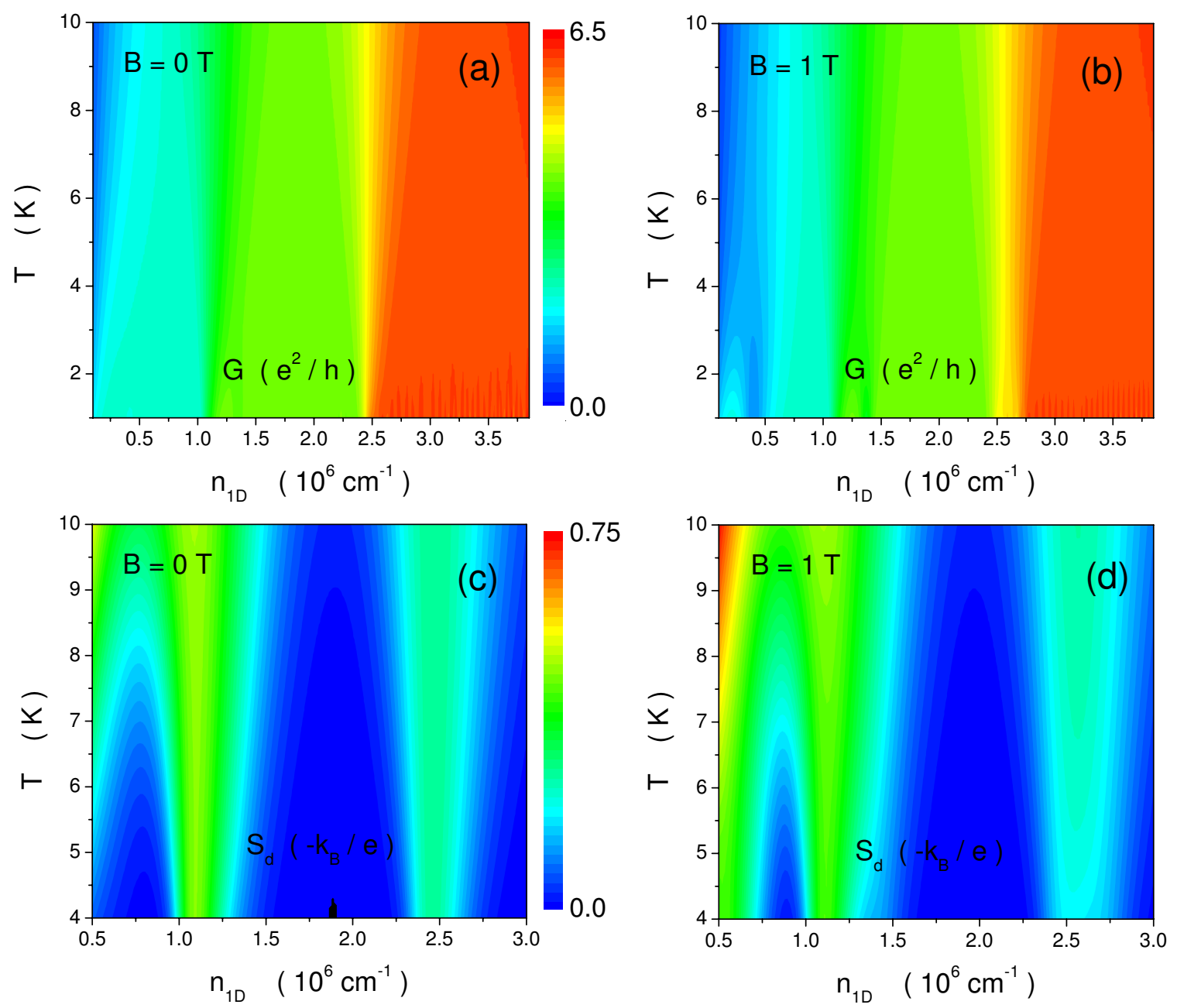

FIG. 5: (Color online) Contour plots of $G$ [in (a) and (b)] and $S_{d}$ [in (c) and (d)] as functions of $n_{1 \mathrm{D}}$ (horizontal axis) and $T$ (vertical axis) for $B=0 \mathrm{~T}$, in ((a) and (c)) and $B=1 \mathrm{~T}((\mathrm{~b})$ and (d)). Here, $V_{b}=0.1 \mathrm{meV}$ and $\tau_{\alpha}=10$. The color scale of $G$ in (a) and (b) varies from 0 (blue) to 6.5 (red), whereas that of $S_{d}$ in (c) and (d) varies from 0 (blue) to 0.75 (red). 

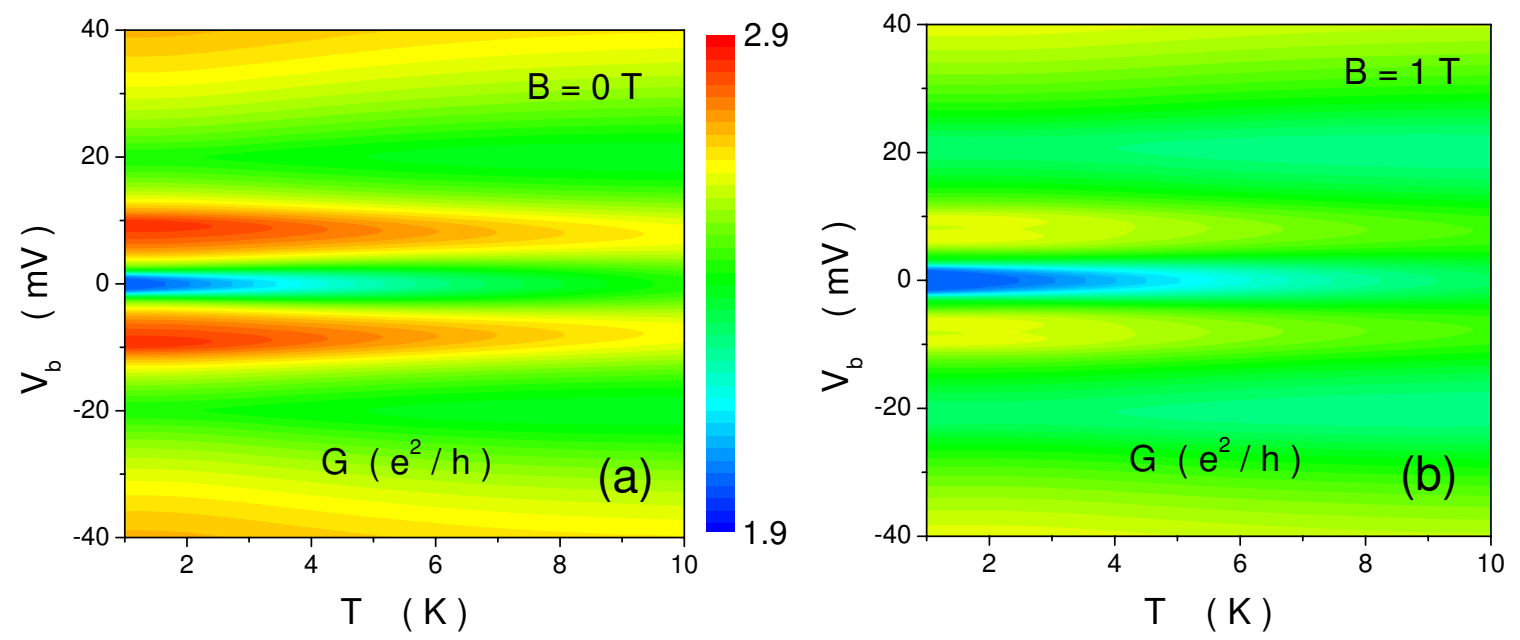

FIG. 6: (Color online) Contour plots of $G$ as functions of $T$ (horizontal axis) and $V_{b}$ (vertical axis) for (a) $B=0 \mathrm{~T}$ and (b) $B=1 \mathrm{~T}$. We chose $n_{1 \mathrm{D}}=10^{6} \mathrm{~cm}^{-1}$ and $\tau_{\alpha}=10$. The color scale for $G$ in (a) and (b) varies from 1.9 (blue) to 2.9 (red).
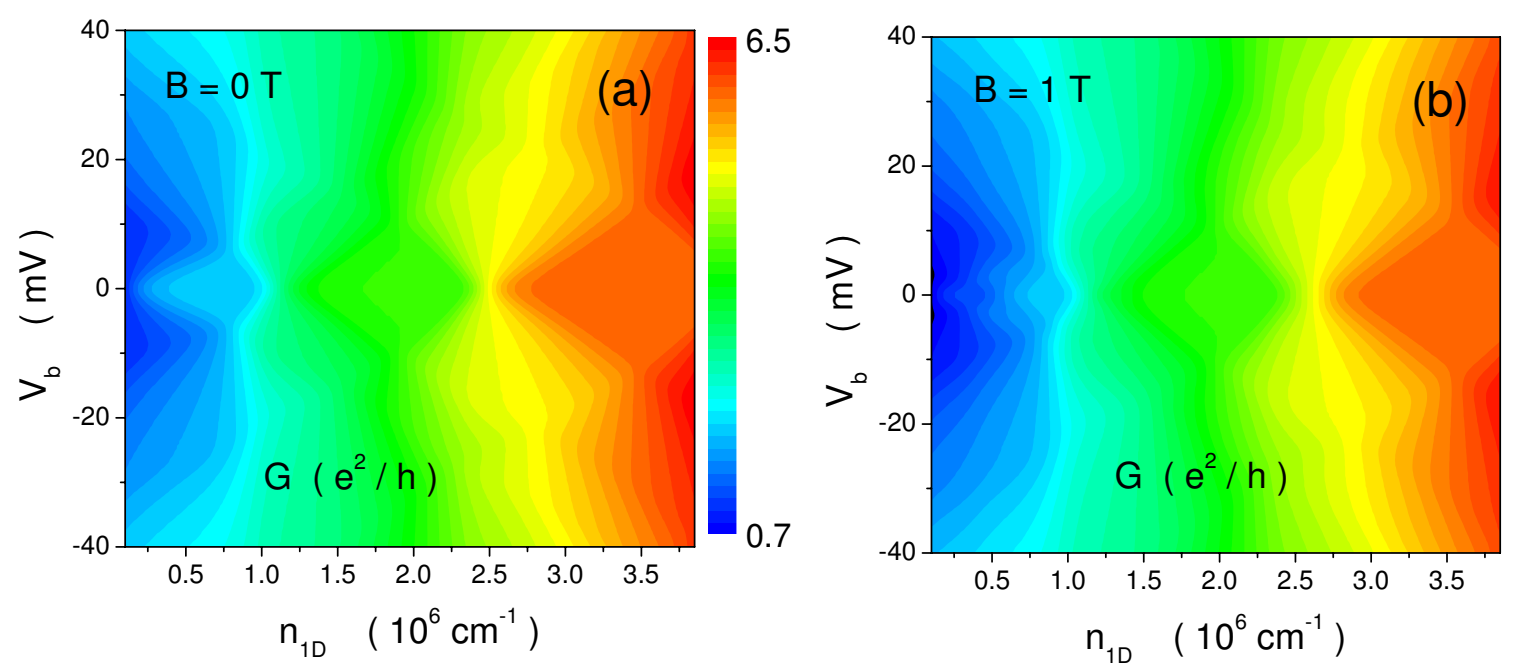

FIG. 7: (Color online) Contour plots of $G$ as functions of $n_{1 \mathrm{D}}$ (transverse) and $V_{b}$ (vertical) for $B=0 \mathrm{~T}$ [in (a)] and $B=1 \mathrm{~T}$ [in (b)]. Here, $T=4 \mathrm{~K}$ and $\tau_{\alpha}=10$. The color scale of $G$ in (a) and (b) is from 0.7 (blue) to 6.5 (red). 